\title{
Fredrika Bremer oder die Tropen zwischen Paradies und Hölle
}

In einem starken Kontrast zu Ida Pfeiffer steht eine schwedische Schriftstellerin, die sich nicht als Reiseschriftstellerin einen Namen machte, sondern als literarische Autorin tout court. Gleichwohl zog sie aber neben ihrer eigentlichen literarischen Tätigkeit durch ihre alleine unternommenen weiten Reisen die nationale wie internationale Aufmerksamkeit auf sich. Darum wollen wir uns in der Vorlesung nun der Schwedin Fredrika Bremer zuwenden. ${ }^{1}$

In der Folge möchte ich Ihnen gerne einige kürzere Passagen aus ihren Reisebriefen aus der Neuen Welt vorstellen. Da ihre Vision der amerikanischen Hemisphäre das Element des Paradieses und damit eine Serie von Topoi aufnahm, mit denen sie sich in eine lange, von Columbus herrührende Tradition einschrieb, und sie freilich auf der anderen Seite die Dimension der Hölle nicht vergaß, welche sie insbesondere durch die Welt der Sklaverei repräsentierte, zeugt ihr reiseliterarisches Schaffen von einem hohen Bewusstseinsgrad im Umgang mit bestimmten literarisch-philosophischen Filiationen der Reiseliteratur, die in unserer Vorlesung im Mittelpunkt stehen. Fredrika war eine nicht nur literarisch, sondern vor allem auch philosophisch hochgebildete Frau. Erlauben sie mir aber zunächst einige biographische Hinweise zu dieser herausragenden Gestalt, ${ }^{2}$ damit wir ihr literarisches Schaffen im Lichte ihres Lebens reflektieren können.

Fredrika Bremer wurde in Finnland, der damals noch schwedischen Provinz, im Jahre 1801 geboren. ${ }^{3}$ Ihr Großvater väterlicherseits hatte sich aus Schweden dorthin begeben und im Handel ein erhebliches Vermögen erwirtschaftet. 1804 und damit noch vor den großen Problemen und dem schließlichen Einmarsch des russischen Zaren in Finnland - waren die Bremers wieder nach Schweden zurückgekehrt und hatten sich in Stockholm niedergelassen, wo sie das Schloss von Arsta erwarben.

1 Vgl. die Vielzahl an Frauenreisen in die Karibik und die auf Fredrika Bremer bezogenen Passagen in Abel, Johanna: Transatlantisches KörperDenken. Reisende Autorinnen des 19. Jahrhunderts in der hispanophonen Karibik. Berlin: Verlag Walter Frey - edition tranvía 2015.

2 Bremer, Fredrika: Durch Nordamerika und Kuba 1849-1851. Stuttgart - Wien: Edition Erdmann 2001.

3 Vgl. hierzu Stendahl, Brita K.: The education of a self-made woman: Fredrika Bremer 1801-1865. Lewiston, N.Y.: E. Mellen Press 1994; Goulard, Matilde: Prólogo. In: Bremer, Fredrika: Cartas desde Cuba. La Habana: Fundación Fernando Ortiz 2002, S. 9-16; sowie Méndez Rodenas, Adriana: En dos tiempos: El viaje de Fredrika Bremer como alegoría de la nación. In: Birkenmaier, Anke / González Echevarría, Roberto (Hg.): Cuba: un siglo de literatura (1902-2002). Madrid:Editorial Colibrí 2004, S. 329-356.

Ә Open Access. (C) 2020 Ottmar Ette, publiziert von De Gruyter. (cc) BY-NC-ND Dieses Werk ist lizenziert unter der Creative Commons Attribution-NonCommericial-NoDerivatives 4.0 Lizenz.

https://doi.org/10.1515/9783110650686-023 


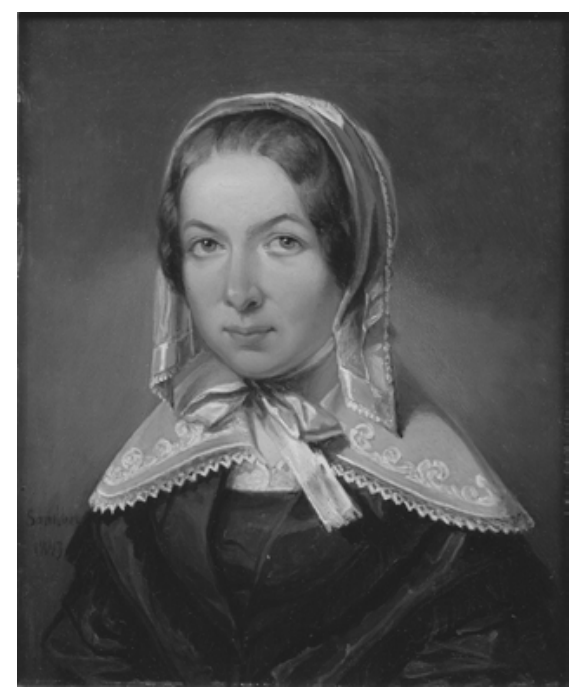

Abb. 80: Fredrika Bremer (Piikiö, 1801 - Gemeinde Haninge, 1865).

Die Bremers bewegten sich zwischen Großbürgertum und Adel, wobei einige Familienmitglieder gewisse adlige Prätentionen besaßen, über die sich Fredrika gerne lustig machte. In ihren ersten Werken, die Alltagsbilder entwarfen, hat Fredrika unterschiedliche Fassungen des Familienlebens der Bremers dargestellt. Als ihr Vater 1830 starb, konnte die Schriftstellerin ihre Erleichterung jedoch kaum verbergen. Ihre Lektüren waren weitgespannt: Sie las etwa Friedrich Schiller und die deutschen Romantiker, liebte Richardson und verfügte über einige Kenntnisse der Soziologie, Philosophie und Theologie. Ihr ganzes Leben lang suchte sie nach einer Religion und Philosophie, denen sie sich anvertrauen konnte.

Mitte der 1830er Jahre, als sie bereits eine literarische Berühmtheit war, setzte sie sich besonders mit der utilitaristischen Philosophie Jeremy Benthams oder John Stuart Mills auseinander, die sie durch ihre Freundschaft mit der Engländerin Frances Eliza Lewin kennengelernt hatte. Im Haus ihrer Schwester Charlotte in Khristianstad im Süden Schwedens traf sie erstmals Per Johan Böklin, der sie in die griechische Philosophie sowie den deutschen Idealismus einführte und zu ihrem großen Lehrmeister wurde. Sie unternahm in der Folge ausgedehnte Studien auch auf dem Gebiet der zeitgenössischen Philosophie, ihre Fourier-Lektüren sind bezeugt. Immer wieder zweifelte sie am Sinn des Romans und suchte in der Philosophie Beistand im Schreiben.

Ihre Romanästhetik entfaltete sich im Spannungsfeld zwischen Romantik und Realismus, wobei sie mit französischen Schriftstellern wie etwa Honoré de 
Balzac bestens vertraut war. In ihren Romanen glänzte sie immer wieder durch präzise Detailbeschreibungen. In ihrem der Romantik verpflichteten Roman Hertha (dt. Hertha oder Geschichte einer Seele. Skizze aus dem wirklichen Leben, 1856/1857) verarbeitete sie viel autobiographisches Material. Zugleich machte sie darin aber auch ihre politischen Überzeugungen sowie ihren Kampf für die Frauenrechte deutlich. Fredrika Bremer wurde als Schriftstellerin zur Initiatorin der schwedischen Frauenbewegung.

Dem Sturm bei der Rezeption ihres Romans entging sie durch eine mehrjährige Auslandsreise, die sie in die Schweiz und nach Italien, aber auch nach Ägypten, Palästina und in die Türkei sowie die Länder des Balkans führte. Ihre Reiseerfahrungen erschienen in dem Band Das Leben in der alten Welt 1860 bis 1863, dem letzten Werk, das Fredrika Bremer veröffentlichte. Sie verstarb 1865 auf dem Schloss ihrer Familie.

Fredrika Bremer wurde nicht zuletzt auch durch ihre journalistischen Texte und Reiseskizzen berühmt, in welchen sie die Probleme ihrer Zeit sehr klar darzustellen verstand. Früh schon war die wohlhabende Fredrika in Europa gereist. Die Erfahrungen ihrer großen Amerikareise hielt sie in ihrem Band Reisen in Nordamerika und auf Cuba fest, wo sie in Briefen an ihre Schwester Agathe ihr zwei Jahre lang dauerndes Durchqueren der Vereinigten Staaten und den Besuch von Gefängnissen oder sozialen Einrichtungen hautnah schilderte. Immer wieder aber beschäftigte sie das Problem der Sklaverei, mit dem sie sich nicht nur im Süden der USA auseinandersetzte, sondern auch auf Kuba, wo sie drei Monate zwischen Februar und Anfang Mai 1851 verbrachte.

Fredrika Bremer kam als reife Frau und ohne Kenntnisse des Spanischen oder der kubanischen Kultur auf die größte der Antilleninseln. Mit ihrem Strohhut und ihrer Zeichenmappe unter dem Arm ging sie durch die Straßen und über die Plätze der Insel, ständig notierend oder zeichnend, indifferent gegenüber dem Aufsehen, das sie als allein reisende Frau erregte. Besonders genau beobachtete Fredrika Bremer das Leben der Schwarzen auf Kuba, ebenso der Haus- und Plantagensklaven wie auch der freigelassenen ehemaligen Sklaven, tief überzeugt von der Unmenschlichkeit der Sklaverei und der Notwendigkeit, gesellschaftliche Veränderungen im Süden der USA wie auf Kuba herbeiführen zu müssen.

Nach dieser kurzen Einführung in das Leben, aber auch einige Werke der schwedischen Autorin wissen wir also bereits, dass wir mit ihrem Bericht über ihre Reise in die USA und nach Kuba ein schönes Exemplum für Reiseliteratur in Briefform vor uns haben. Diese Form hatten wir im 18. Jahrhundert bereits bei Bernardin de Saint-Pierre kennengelernt und festgestellt, dass sie natürlich recht nahe an jener des 'klassischen' Reisetagebuches ist. Doch die Form des 
Briefes ermöglicht eine noch stärkere subjektive Brechung der dargestellten Erlebnisse und Ereignisse, wobei es gerade dieser Effekt ist, den Fredrika Bremer in ihrer romantischen Konzeptionen zuneigenden Literaturauffassung im Auge hatte. Zugleich bietet der (asymmetrische) Dialog die Möglichkeit, eine Spiegelung des Dargestellten im Bewusstsein der Adressatin oder des Adressaten vorzunehmen. Diese Charakteristika der gewählten reiseliterarischen Form nutzte Fredrika Bremer weidlich aus.

Der erste dieser auf Kuba bezogenen Briefe, die sich in das große AmerikaWerk einreihen, ist bereits der einunddreißigste Brief. Er stellt, datiert auf Havanna den 5. Februar 1851, die Überfahrt von New Orleans nach Havanna dar. Sie können sich jetzt bereits vorstellen, welche Szenerie nun folgt. Denn ich habe in der Tat die Texte (und auch die AutorInnen) so ausgewählt, dass wir immer wieder an denselben geographischen Stellen 'vorbeischauen' und vergleichen können, wie die Perspektiven und Auswahlkriterien jeweils repräsentativ für die ausgewählten Texte sind. So haben wir hier also erneut eine Einfahrt in den Hafen von Havanna vor uns, das Tor zur Insel Kuba und zugleich jener Ort, an dem man gleichsam in einer mise en abyme die gesamte Insel bereits porträtieren kann.

A la mañana siguiente entramos en el puerto de La Habana.

Las olas se levantaban y rompían furiosamente contra el saliente cabo donde la fortaleza de El Morro se levanta con sus muros y torres (una de ellas muy alta) y defiende la angosta entrada al puerto. Pero en la bella bahía, casi circular, estábamos tan en calma como en el más tranquilo estanque, y el sol lucía sobre un mundo de objetos nuevos en torno mío.

Allí se extendía la gran ciudad de La Habana, a lo largo de la costa, a la derecha según se entra al puerto, con casas bajas de todos los colores: azules, amarillas, verdes, anaranjadas, como un enorme depósito de cristales abigarrados y objetos de porcelana en una tienda de regalos; y ningún humo, ni la menor columna de humo daba indicios de la atmósfera de una ciudad, con la vida de las cocinas o de las fábricas, como yo estaba acostumbrada a ver en las ciudades norteamericanas. Grupos de palmeras se elevaban entre las casas.

Una altura a nuestra izquierda estaba cubierta con multitud de plantas extrañas, semejantes a altos candelabros verdes con muchos brazos. Entre las colinas verdes que se veían alrededor del puerto había grupos de casas de campo, y bosquecillos de cocoteros y otros árboles del tipo de las palmeras; y sobre todo esto se extendía el cielo más claro y suave, y se respiraba el aire más delicioso. El agua del puerto parecía clara como el cristal, y el aire y los colores eran de la más diáfana claridad y serenidad. Entre los objetos que me llamaron la atención se destacan la fortaleza donde están encerrados los prisioneros, otra prisión y ... la horca. Pero las bellas palmeras ondulantes, las verdes colinas, encantaron mi vista. ${ }^{4}$

4 Bremer, Fredrika: Cartas desde Cuba. Herausgegeben von Redy Puebla Borrero. Übersetzung von Matilde Goulard de Westberg. La Habana: Fundación Fernano Ortíz 2002, S. 21 f. 
In dieser literarisch brillanten und wohlkalkulierten Passage fällt auf, dass wir es in der reiseliterarischen écriture von Fredrika Bremer mit einer Kontrasttechnik zu tun haben, die freilich an der Landschaft und der Gestaltung des Hafeneinfahrtsbereiches ausgerichtet ist und mit den sozialen wie politischen Gegensätzen der Insel Kuba noch ein wenig hinter dem Berg hält. Doch auch in diesem Bereich zeichnet sich bereits einiges schematisch und umrisshaft ab.

In einer ersten Annäherung brechen sich die Wellen wild an der Festung des Morro, der über die Einfahrt von Havanna wacht. Dies ist im Grunde recht gemeinplatzartig, denn Festungen pflegen sich wild und uneinnehmbar aus der sie umgebenden Landschaft aufzutürmen. Freilich wird dieses Element später wiederaufgenommen und leitet dann - nach der hier abgedruckten Passage - über zu einer Intervention der spanischen Kolonialbehörden, welche die Passagiere nicht von Bord lassen wollen, weil ein ausgemachter Gegner des Kolonialregimes, ein US-Amerikaner, der bei der berüchtigten Expedition von Narciso López mit im Spiel war, ebenfalls den Hafen von Havanna, allerdings auf dem Weg nach Kalifornien, besucht. Die noch zu Spanien gehörige Insel Kuba ist schon zum damaligen Zeitpunkt ein Spielball der mächtigen Interessen des auf Expansion bedachten Nachbarn USA.

Im Gegensatz zu diesen wenigen dunkleren Tönen freilich finden wir ein sehr zartes, fein gemaltes buntes Bild, das zunächst die Stadt Havanna wie frisch aus dem Spielzeugladen kommend, mit allerlei bunten Häuschen besetzt, in den Blick der Reisenden rückt. Das Kinderspielzeug ist harmlos, unschuldig, offen - und zugleich so ganz anders als die Städte Nordamerikas, aus denen es überall pufft und qualmt, kocht und brodelt. Damit wird zum einen die wunderbare Luft, die Fredrika Bremer so wichtig ist, argumentativ begründet, aber auch ein Hinweis gegeben auf eine fehlende Industrialisierung, eine fehlende wirtschaftliche Modernisierung dieser Stadt, die eben nicht so geschäftig daherkommt wie die industriellen Städte der USA. Bremer führt damit einen wichtigen ökonomischen und soziopolitischen Gegensatz zwischen der Insel Kuba und den Vereinigten Staaten von Amerika ein - und sie tut dies äußerst geschickt anhand eines kleinen Details im Blick der Reisenden bei der Einfahrt in den Hafen von Havanna.

Gewiss hat dies auch eine paradieshaft-unschuldige Seite, die vor allem beim Blick über die Bucht von Havanna sichtbar und anschaulich wird. Fredrika Bremer benutzt die Tropen des Diskurses über die Tropen und spielt auf dieser Klaviatur durchaus meisterlich. Sah Alexander von Humboldt dieselbe Bucht voller Segelschiffe und ihrer Masten, welche einen wahren Wald bilden und die natürlichen Stämme der Palmen gleichsam verlängern (und zugleich die Gefahren der Stadt verbergen), so findet Fredrika Bremer am selben reiseliterarischen Ort der Ankunft überall eine wunderbare Harmonie von Natur und 
Mensch vor, in welche sie eintaucht. Denn mitten in einer ausgedehnten Tropenlandschaft, die von Palmen überragt wird, haben sich die schönsten Landhäuser angesiedelt, die eine friedliche, ruhige Bucht umsäumen. Das Bild eines wahren irdischen Paradieses entsteht.

Dass Kuba im weiteren Fortgang des Briefes - vor allem unter dem Vorzeichen der Sklaverei - auch die andere Seite der Medaille, nämlich die Hölle auf Erden repräsentieren kann, wird gegen Ende der hier zitierten Stelle in Ansätzen deutlich. Mit dem Verweis auf die Gefangenen - die Festung des Morro diente lange Zeit als das berüchtigtste Gefängnis der kolonialspanischen Behörden in den Amerikas -, auf die Zuchthäuser und auf den Hinrichtungsort der horca, des Galgens, welcher auf die noch alles beherrschende kolonialspanische Macht verweist, rückt unverkennbar ein anderes, gewalttätiges Element ins Bild, das der Leserin zumindest schon einmal zur Kenntnis gebracht wird, aber noch im Hintergrund verbleibt.

Vordergründig aber geht es um die Darstellung eines locus amoenus, für den es - soweit das Inventar - grüne Wiesen und Hügel, ein klares, helles Wasser, eine wunderbare Luft und eine sonnige Atmosphäre braucht. Auch wenn wir hier kein klares Bächlein haben, das lustig vor sich hinplätschert, und keine Vögel, die munter für uns zwitschern und singen, ist doch unverkennbar, dass Kuba vor den Augen der Schwedin noch immer mit jenem Zauber und Charme vorbelastet ist, der schon in den Augen Cristóbal Colóns dreieinhalb Jahrhunderte zuvor die Insel zu einem Lustort machte.

Doch am reiseliterarischen Ort der Ankunft in der Zielregion haben wir es mit einem fraktalen Bildnis der gesamten Insel Kuba zu tun. Die mise en abyme impliziert freilich die zumindest angedeutete, wenn auch nicht breit ausgeführte Hintergrundfolie menschlicher und kolonialer Herrschaft und Gewalt.

Nicht einmal das sechsstündige Warten der Passagiere an Bord auf das Verlassen des Schiffes konnte Fredrika Bremer, so schrieb sie in ihrem literarischen Brief an ihre Schwester Agathe, vom Zauber abhalten, den die Insel - und all die neuen Gegenstände, die natürlich nur für die Schwedin neu waren, aber an die Bezeichnung der Neuen Welt erinnern - auf sie ausübte. Kuba hat sie also von Beginn an ganz ergriffen.

Das hintergründige Spiel von Paradies, Sündenfall und Hölle findet sich immer wieder in den Beschreibungen der Insel Kuba und ihrer Phänomene. Dieses Motiv, geradezu ein Leitmotiv ihres Reiseberichts, klang ja bereits gleich zu Beginn der Einfahrt in den Hafen von Havanna an, und es zieht sich - nicht nur in jenen Briefen, die sich ausführlich der Sklaverei widmen - durch den gesamten Bericht. Ich darf bereits an dieser Stelle hinzufügen, dass sich daran bis heute nicht viel geändert hat. Denn auch das heutige, das revolutionäre Kuba Fidel Castros und seiner Nachfolger, ist noch immer von einem grundlegenden 
Zwiespalt geprägt, der immer wieder um neue Facetten bereichert wird, letztlich aber immer um die Pole Paradies und Hölle, Insel-Freiheit und Insel-Gefängnis, oszilliert.

So ist auch Kuba heute für die einen ein Paradies, entweder im sozialistischen oder rein sonnigen Sinne, und für die anderen eine Hölle, was im allgemeinen politisch verstanden wird. Hier hat sich eine Ambivalenz quer zu allen politischen und kulturellen Veränderungen in der stereotypen Darstellung der Größten der Antilleninseln fortgesetzt.

Aber diese Bilder sind keineswegs neu, wie wir von Fredrika Bremer erfahren:

He oído muchas quejas sobre la administración de la isla, sobre los monopolios, las injusticias, los robos cometidos en todas partes, tanto por los funcionarios como por los juriconsultos. Se dice expresamente que devoran 'la parte de las viudas y de los huérfanos'. Sobre este punto he oído historias casi increíbles. Ahora se fijan muchas esperanzas en el nuevo gobernador general Concha, que fue enviado por España hace dos meses, y quien, según dicen, es un hombre bueno y honrado. El gobernador recientemente depuesto se hizo famoso por sus fraudes, que lo convirtieron en un hombre rico. Se cuenta que el clero es bastante poco religioso, que la mayoría vive en opuesta contradicción con sus votos, y se asegura que la religión ... ha muerto. Sigue habiendo trata de esclavos, aunque ocultamente. La administración lo sabe, pero recibe treinta o cincuenta pesos por cada esclavo que es traído de Africa, cierra los ojos ante el tráfico y hasta lo favorece, según se dice.

¡Ay, que este paraíso terrestre haya que estar siempre envenenado por la vieja serpiente! $!^{5}$

Interessant ist, dass Fredrika Bremer, die sich sehr stark in den Kreisen relativ begüterter Ausländer aufhält und Engländer, Deutsche, Franzosen und auch Schweden in Havanna trifft, von den politischen Verhältnissen gleichsam nur vom Hörensagen berichtet. Sie gibt gleichsam zu Protokoll, was ihr zu Ohren gekommen ist. Als eigentliche Augenzeugin tritt sie in ihren Briefen auf dem Feld des Politischen und der kolonialspanischen Verwaltung kaum einmal auf.

Damit besitzt sie als Stimme auf diesem Gebiet eine nur eingeschränkte Legitimität, auch wenn die Vorherrschaft des Auges als Informationsquelle über das Ohr zu ihrer Zeit noch nicht so radikal ausgeprägt war wie in einer gewgenwärtigen Epoche dominant visueller Informationsübermittlung. Im 19. Jahrhundert war das Hörensagen noch nicht so negativ eingefärbt wie in unseren Tagen, wo man sich nur selten auf das Gehörte verlässt und lieber den eigenen Augen beziehungsweise der Schrift glaubt, und stammt sie auch aus den sogenannten sozialen Medien.

In der obigen Passage wird zunächst die Korruption der Verwaltung angeprangert, die vom Gobernador angefangen auf die rasche eigene Bereicherung

5 Bremer, Fredrika: Cartas desde Cuba, S. 36. 
hin ausgelegt ist. Aber auch der katholische Klerus bekommt sein Fett weg, erscheint er doch nicht als religiös, sondern als hochgradig korrupt, mit der Macht und deren Interessen verfilzt. Übrigens wird dies im 20. Jahrhundert dann dazu führen, dass die Katholische Kirche in Kuba immer weniger Anhänger im Lande finden wird und gänzlich an Legitimität einbüßt. Erst im Verlauf der letzten Jahrzehnte haben sich die Dinge unter der Revolution, die zuvor einen völlig kirchenfeindlichen Kurs fuhr, verändert - und zwar bereits schon geraume Zeit vor dem berühmten und öffentlichkeitswirksamen Besuch des Papstes. Daher gab und gibt es gute Gründe dafür, dass sich im Verlauf der zweiten Hälfte des 19. und dann vor allem in der ersten Hälfte des 20. Jahrhunderts verschiedene afrokubanische Kulte wesentlich stärker entwickeln konnten und heute eine selbst von der Revolution nicht leicht $\mathrm{zu}$ kontrollierende Machtposition einnehmen.

In der Zeit von Fredrika Bremer aber war das eigentliche Übel die Problematik von Sklavenhandel und Sklaverei, die natürlich auch in den Südstaaten der USA vorherrschte. Der Handel mit Sklaven, das hatten wir schon am Beispiel Brasilien gesehen, bestand fort. Zwar hatte Spanien auf langanhaltenden Druck Englands, das nicht nur aus philanthropischen, sondern auch aus handfesten kommerziellen Interessen handelte, sich vertraglich verpflichtet, auf den weiteren Transport, mithin auf die fortgesetzte Deportation von Sklaven aus Afrika auf die Antillen zu verzichten.

Doch die Wirklichkeit war eine andere: Englische und andere Schiffe waren ständig damit beschäftigt, die Küsten von Afrika, Kuba, Puerto Rico oder Brasilien abzusuchen, um den florierenden Schleichhandel mit Sklaven zu unterbinden.

Bei den nicht selten dramatischen Verfolgungsjagden waren die Schwarzen oftmals nicht die Geretteten, wurden sie doch nicht selten einfach über Bord geworfen, so dass die Besitzer von Sklavenschiffen straflos ausgingen. Fredrika Bremer hatte richtig gehört: Die spanische Kolonialverwaltung auf Kuba widersetzte sich allen Versuchen, den Sklavenhandel effizient zu unterbinden, zumal ihr die ansässigen Kreolen und anderen Plantagenbesitzer vorrechneten, dass dies noch immer die beste und billigste Möglichkeit wäre, wohlfeile Arbeitskräfte für die kubanische Produktion und insbesondere die Erzeugung von Zucker zu generieren. Daran verdiente die spanische Verwaltung auf Kuba sehr gut, wie auch Fredrika Bremer sehr wohl wusste.

An diesem Punkt also geschieht der Sündenfall im irdischen Paradies; und die von Fredrika Bremer evozierte Schlange ist hier ganz offensichtlich ein unmenschliches Gewinnstreben innerhalb eines kolonialen kapitalistischen Systems, das in so starkem Kontrast zum Landschaftsbild, dem Klima und der herrlichen Luft auf der Insel steht. Denn dieses Klima, so die schwedische Autorin, habe die Indianer früher so sanft gemacht, und so seien auch die heutigen 
Kreolen im Grunde sehr sanfte, gutmütige und wohltätige Menschen. Mag sein, dass Fredrika Bremer davon wirklich überzeugt war.

Die schwedische Schriftstellerin war eine ausgezeichnete Beobachterin, die sich oft mit ihrem Zeichenblock allein aufmachte, um bestimmte Pflanzen, Landschaften oder andere Eindrücke festzuhalten. Nicht selten merkt man ihr diesen visuellen Gestus, diese Betonung des Optischen, sehr an. Sie bewegte sich, beobachtete aber auch viel in den Häusern, und dabei notierte sie sich vieles nicht zuletzt zur Situation der Frauen auf Kuba, die sie als Frauenrechtlerin kritisch sah. Da sie vor allem im gut situierten Handelsbürgertum verkehrte, bezogen sich ihre Beobachtungen vor allem auf Frauen, die sich entweder zumindest eine Köchin oder gar einen Koch und Einkäuferinnen halten konnten. Die sogenannten Hausfrauen mussten sich um hausfräuliche Pflichten nicht kümmern, eine Tatsache, die Fredrika Bremer überrascht konstatierte.

Und nicht weniger überrascht notierte sie, dass sich die schwarzen Sklavinnen und Sklaven gerade bei diesen Aufgaben hervorragend bewährten, wenn sie den Küchenzettel zusammenstellten und für alles - oftmals ohne jede Aufsicht - sorgten. Gertrudis Gómez de Avellaneda, die große Dichterin des kubanischen (und spanischen) 19. Jahrhunderts, belächelte die Spanierinnen, die sich mit Hausarbeiten und mit Putzen abgeben mussten, während die Kubanerinnen frei über ihre Zeit verfügten. Die Dichterin berichtete stolz, dass sich kubanische Frauen - weiße Frauen, wohlgemerkt - mit so schönen Dingen wie Kunst, Literatur und Musik beschäftigten, da sie keine Wohnzimmer säubern, Socken stopfen oder in der Küche hantieren mussten. Denn in Kuba gab es für diese niederen Arbeiten genügend Sklavinnen und Hausangestellte.

Eben dieses Phänomen beobachtete Fredrika Bremer sehr genau. Aufmerksam war die an einer Emanzipation der Frauen ausgerichtete schwedische Schriftstellerin schließlich auch noch auf einem dritten Feld, dem der Kultur der schwarzen und mulattischen Bevölkerung, deren Musik und Tänze sie sich mehrfach aus der Nähe anschaute. Wir hatten schon gesehen, wie auch Ida Pfeiffer von diesem Thema fasziniert war. Fredrika Bremer freilich wird diesen kulturellen Praktiken sehr viel gerechter. Ich möchte Ihnen hier einen Auszug aus einem Brief an die Hand geben, der auf den 12. Februar 1851 datiert ist. Darin geht es um die kulturellen Ausdrucksformen und kulturellen Praktiken der schwarzen kubanischen Bevölkerung.

Al mediodía, escuché desde varios puntos el ritmo vivo del tambor africano, no muy diferente del ruido que hacen los trillos en las granjas de nuestro país; sólo que aquí hay una vida mucho más animada. Era la señal de que los negros libres tenían sus bailes en los lugares de reunión de la comarca. Mi anfitrión tuvo la amabilidad de acompañarme a uno de éstos, muy cerca de nuestro Cerro. Allí, en una habitación parecida a la gran sala de una hostería de nuestro país, vi a tres negros, desnudos de la cintura para arriba, con 
figuras y rostros enérgicos y salvajes, golpeando los tambores con una animación igualmente enérgica. Los tambores estaban hechos de troncos de árboles huecos, con una piel tensa encima. Los negros golpeaban la piel tensa, en parte con palillos y en parte con las manos - pulgares y palmas-, con una habilidad maravillosa, una perfección artística salvaje, o, más bien diría, un arte natural perfecto. Golpeaban los tambores como la abeja zumba o el pájaro canta o el castor construye su vivienda. Compás y ritmo, que a veces cambiaban, eran extraordinarios. No se puede imaginar una energía animada más perfecta en su naturalidad y en el compás desigualmente igual. Mantenían los tambores entre las rodillas. En las muñecas llevaban grandes esferas, llenas de piedrecillas u otros objetos que sonaban, decoradas por el exterior con manojos de plumas de gallo. Lo principal parece que era conseguir todo el ruido posible. Había algunas parejas que bailaban, damas de diferentes tonos de color, enfundadas en harapientos atavíos y tocadas con adornos de colores chillones, y hombres (negros) sin adornos y casi sin ropas en la mitad superior del cuerpo. Un hombre tomó a una mujer de la mano y comenzaron a bailar. Ella giraba sobre un mismo lugar con los ojos bajos; él daba vueltas a su alrededor con una gran cantidad de cabriolas tiernas; entre ellas, las volteretas y saltos más exaltados imaginables, que eran dignos de admiración por su audacia y agilidad. ${ }^{6}$

Die Auswahl der Kulturpraxis des Tanzes bietet Ihnen an dieser Stelle einen leicht gemachten Vergleich mit unserer Ida Pfeiffer. Bei Fredrika Bremer bemerkt man einen geradezu professionellen anthropologisch-ethnologischen Blick auf diese Elemente einer Volkskultur, einer cultura popular, für welche die Schwedin offenkundig schon beim ersten Besuch eine große Bewunderung empfindet. Man merkt ihrer Beschreibung in jeder Zeile an, dass sie das Gesehene und Gehörte tief beeindruckt.

Dabei sind die Beschreibungskriterien und die diskursiven Mechanismen durchaus zwiespältig. Denn Bremer bringt das Trommeln der Schwarzen nicht selten mit dem Agieren von Tieren in Verbindung, eine Tatsache, die uns darauf hinweist, dass hier die volkskulturellen Praktiken zweifellos in Richtung 'wilder Natur' aus der Kultur ausgebürgert zu werden scheinen. Doch diese auf den ersten Blick rassistische Bewegung des Ausschlusses wird sehr stark konterkariert durch eine gegenläufige Bewegung, die diese kulturellen Praktiken durchaus in den Kontext von Kunst und Kultur rückt.

Die beiden Adjektive, die in ein spannungsvolles Verhältnis eintreten, sind 'wild' und 'künstlerisch' - eine ebenso paradoxe Konstruktion wie der Hinweis Bremers auf den ungleich gleichen Rhythmus, den „compás desigualmente igual“. Die europäische Beobachterin hat offenkundig Schwierigkeiten, Kategorien der Einordnung zu finden, ist aber sehr wohl bereit, sich auf eine präzise Beschreibung einzulassen. In der Tat erleben wir einen Tanz der freien Schwarzen und Mulatten mit, der in gewisser Weise eine Vorläuferform dessen darstellt,

6 Bremer, Fredrika: Cartas desde Cuba, S. 40 f. 
was sich nach der Wende zum 20. Jahrhundert dann als Vorfahren des kubanischen Son und der lateinamerikanischen Salsa herausbilden sollte. Die kubanischen contradanzas aber waren gerade für europäische Zeitgenossen kulturelle Praktiken, die extrem weit von Tanz und Musik in Europa entfernt waren.

Fredrika Bremer beweist hier aber eine nicht nur präzisere Beobachtungsgabe als Ida Pfeiffer, sondern zugleich auch ein offenes Verständnis für derartige kulturelle Ausdrucksformen. Im Übrigen gelingt es ihr auch in diesem Auszug, das Geschlechterverhältnis im Tanz nachzuzeichnen, eine Tatsache, die ihr übrigens gleich mehrfach, noch an anderen Stellen ihrer Reisebriefe, gut von der Hand ging. Entscheidend aber ist, dass das charakteristische Oszillieren zwischen Kunst und Wildheit, aber auch die Präsenz halbnackter männlicher Körper, mit Blick auf die sie - übrigens ähnlich wie Ida Pfeiffer - von Herkulessen sprach, sehr stark faszinierten. Auch bei Fredrika Bremer kann man mit Fug und Recht von der Präsenz eines Herkules-Motivs sprechen.

Dass sie von der Schönheit der kubanischen Frauen nicht allzu sehr angetan war, sollte die Schwedin auch noch später bei anderen Beschreibungen äußern. Wir merken daran, dass die europäischen Schönheitskategorien noch nicht hin zur Aufwertung bestimmter mulattischer und schwarzer Körperformen tendierten, wie sie uns nicht zuletzt durch die Werbung heute so vertraut sind. Gleich zu Beginn ihres Kubaaufenthaltes hatte Fredrika Bremer nicht ohne Stolz notiert, dass sich mit der Schönheit und Ausstrahlungskraft der Europäerinnen nichts vergleichen lasse. Die Abwertung der Körperformen und der Ästhetik schwarzer Frauen ist bei ihr weitaus weniger ausgeprägt als bei Ida Pfeiffer, kommt aber vor allem dadurch zum Ausdruck, dass sie die Europäerinnen besonders gerne kontrastiv in ihrer Schönheit hervorhebt. Doch beeindrucken ihre anthropologisch recht exakten Darstellungen von Musik- und Tanzformen sowie Choreographien der freien schwarzen und mulattischen Bevölkerung auf Kuba: in einem kolonialen Sklavenhalterstaat, der immerhin derartige Vergnügungen bisweilen einräumte.

Fredrika Bremer unterstrich aus ihrer als philanthropisch zu bezeichnenden Haltung heraus diese relative Großzügigkeit der spanischen Leyes de Indias; und sie beobachtete das Leben der Schwarzen im Grunde mit großem Wohlwollen. Dies hält sie freilich nicht davon ab, bisweilen Bemerkungen über die schwarze oder mulattische Bevölkerung einzustreuen, die wir heute ganz eindeutig als rassistisch bezeichnen würden. Bei einem Ball der freien Schwarzen in der Stadt Matanzas etwa beschreibt sie ausführlich das gesamte Fest, widmet sich dann aber der besten Tänzerin und sagt von ihr, sie sei so hässlich, dass sie trotz ihrer schönen Kleider nach französischer Mode aussehe, als sei sie ein in ein schönes Kleid gesteckter Affe. Im Übrigen sei der gesamte Ball durchaus etwas, das man als zivilisiertes Leben der Schwarzen bezeichnen könnte, wären die Sitten nicht so formell und so aufgesetzt. 
In derlei Passagen zeigt sich ebenso wie in der Frage der Schönheit, wie tief auch bei wohlmeinenden Europäerinnen und Europäern die Vorurteile gegenüber der abgewerteten schwarzen Bevölkerung zum damaligen Zeitpunkt - und sicherlich auch später - waren. Denn mit Fredrika Bremer haben wir es ja zweifellos mit einer Nordeuropäerin zu tun, die sich eindeutig gegen die Sklaverei ausspricht, sich für eine breitere geistige Entwicklung der Schwarzen und Mulatten einsetzt und sich im übrigen - wie auch ihr eigenes Leben zeigt - deutlich für eine Selbstbestimmung der Frauen stark macht, so dass sie später auch zeitweise als Galionsfigur des schwedischen Feminismus und erster Vereinigungen von Frauen dienen konnte.

Freilich zählen auch die kritischen Äußerungen Fredrika Bremers zu jener Art von Reiseberichten, die sozusagen nicht nur eine Reiseroute, sondern auch einen Weg der jeweiligen subjektiven Befindlichkeit der Reisenden mitzuteilen versuchen. Die Spannung zwischen dem Ich und dem von ihm beschriebenen Objekten überträgt sich auf das Lesepublikum. Gerade hierin zeigen sich die spezifischen Möglichkeiten des Briefes innerhalb der Gattung der Reiseliteratur Subjektivität zu erzeugen, Identifikationsprozesse einzuleiten und gleichsam die Leserschaft 'direkt' und unvermittelt anzusprechen. So entsteht ein Eindruck von Authentizität.

Die Briefe aus Kuba sind an die Schwester Agathe in der Heimat gerichtet. Und doch finden wir relativ wenig Schwesterliches: Fredrika Bremer - dies machen ihre Äußerungen immer wieder deutlich - bevorzugt es, sich allein auf vor ihr von Frauen wenig oder nie begangene Wege zu begeben. Bezüglich ihrer Reiseplanung hat sie ähnlich wie Ida Pfeiffer ihren ganz eigenen Kopf.

Dies zeigt sich gerade auch bei ihrer Kubareise, selbst wenn man dort anmerken muss, dass sie als Frau ganz selbstverständlich über eine Reihe von Vorteilen gegenüber Männern verfügt. Selten kommt sie einmal dazu, ihre eigenen Rechnungen zu begleichen, zu groß ist die Gastfreundschaft und zu häufig sind kleine und große Geschenke, die man ihr darbringt, bisweilen ohne den Namen der oder des Beschenkenden zu nennen. Die Briefe aus Kuba bilden innerhalb der Amerikareise gleichsam eine Reise in der Reise; und sie weisen zugleich auch auf einen Zyklus, ein Kreisschema hermeneutischer Bewegungsfiguren hin, das diesen Reiseteil als relativ unabhängigen Bestandteil der gesamten Reise ausweist. Diese Verbundenheit und Autonomie trägt zum Zauber dieser kubanischen Briefe bei.

Von dieser subjektiven weiblichen Position aus werden freilich immer wieder - und gerade auch gegen Schluss des Berichtes - Versuche unternommen, die Gesamtsituation auf Kuba zu erfassen. Damit schließt sich gleichsam der Kreis der Reise, beziehungsweise der hermeneutischen Kreisstruktur, innerhalb einer größeren Kreisstruktur zwischen Alter und Neuer Welt. Die Argumentation 
der Schwedin kommt hierbei $\mathrm{zu}$ einem Abschluss und zugleich $\mathrm{zu}$ einem Zirkelschluss.

Ich möchte Ihnen dies gerne anhand ausgewählter Teile der letzten Seiten des sechsunddreißigsten und letzten Briefes aus Kuba, der unmittelbar vor der Abfahrt verfasst wurde, vorführen:

Durante mis paseos por La Habana he tenido siempre el placer de contemplar a la población negra, que me ha parecido más libre y más feliz que en las ciudades de los Estados Unidos. Aquí se ve, más a menudo que allí, a los negros y a los mulatos ejerciendo el comercio, y sus mujeres, por lo general, están muy bien vestidas y son elegantes. En las espléndidas calles se ven, no pocas veces, a mulatas con flores en el cabello y con sus familias, paseándose en una forma que denota bienestar y libertad. [. . .]

Parece que los negros cristianos libres permanecen muy fieles a la mentalidad que tenían en Africa. Les han construido grandes casas con habitaciones cómodas, cocina, y huertos, de modo que en cuestión de vivienda y vida de trabajo puedan tener al mismo tiempo todas las ventajas de la vida privada y de la asociación. ¡Es inútil! Las grandes y cómodas casas de piedra se han quedado vacías. Al negro no le gustan ni las casas de piedra ni las asociaciones. La finalidad primaria de cada negro es poderse comprar un lote de tierra propia [...] donde poder construirse un bohío de cortezas de árbol cubierto con guano, plantar los árboles de su país natal, y cultivar en un pequeño terreno caña de azúcar, o maíz y viandas. Trabaja para conseguir ese paraíso terrenal. Una vez llegado a eso, su ideal es descansar, gozar lo más posible y trabajar lo menos que pueda. ¿Y para qué había de trabajar? La ambición y el deseo de saber, de poseer el mundo espiritual y materialmente, que el Creador puso en la raza caucásica, no se los concedió a él. En cambio recibió la capacidad del goce despreocupado, del alegre carácter, del canto y la danza rítmicos. Las latitudes bajo las que él nació favorecen estos últimos dones y se oponen a los primeros. [. . . ]

He echado una última ojeada a Cuba desde la azotea de la casa de Alfredo Sauval. Fue ayer por la tarde, durante la puesta del sol. Por última vez he visto sus bellos palmares, sus abigarradas y relucientes casas, su suave cielo, su mar azul claro a esa luz, en ese aire encantador y mágico del anochecer. Hoy por la tarde me embarco en el 'Isabel', y le digo adiós para siempre a las palmeras y a las ceibas de Cuba, a los cocuyos y a las contradanzas, a las guardarrayas y a las constelaciones, a los tambores africanos, a las canciones y a los bailes, a este pueblo feliz y desgraciado, ja su infierno y a su paraíso! [...] He aspirado una nueva vida en Cuba, pero vivir aquí no podría. ¡Esto sólo podría hacerlo donde exista y crezca la libertad! ${ }^{7}$

In dieser langen Passage wird gleichsam sehr bewusst der argumentative Kreis geschlossen. Noch einmal erscheinen alle eingangs neuen Gegenstände ein letztes Mal vor dem inneren Auge der Reisenden und damit vor dem Auge des Lesepublikums: wie in einem Bilderreigen, der sich öffnet und dann schließt. Zentral ist dabei die Frage der Schwarzen und insbesondere der freien Schwarzen: Auf diesem Feld wird ein insgesamt positives Fazit auch gerade im Vergleich mit den

7 Bremer, Fredrika: Cartas desde Cuba, S. 192-196. 
Südstaaten der USA gezogen, gebe es auf Kuba doch für freie Schwarze weitaus bessere Lebensmöglichkeiten als in den Sklavenstaaten des Südens der USA.

Die Vereinigten Staaten bilden für die Schwedin den Kontext der Vergleichsmöglichkeiten, und zwar zu einem Zeitpunkt, als man insbesondere im Süden der USA die Pläne forcierte, Kuba von Spanien abzukaufen und damit die Sklaverei auch auf den dann karibischen Teil der USA auszuweiten, um dergestalt das Gewicht der Sklavenstaaten insgesamt zu vergrößern. Die schwedische Reisende war sich dieser Zusammenhänge durchaus bewusst.

Aufschlussreich ist es aber auch, dass Fredrika Bremer eine vom Schöpfergott gleichsam vorgenommene Scheidung zwischen der kaukasischen Rasse, also der Rasse der Weißen - eine Scheidung, die seit Ende des 18. Jahrhunderts endgültig durch die Lehren eines Blumenbach und vieler anderer befestigt war und in den USA bis heute gilt - und den anderen Rassen vornimmt. Sie übernimmt dabei die gängigen (pseudowissenschaftlichen) Einteilungen ihrer Zeit.

Den Vertreterinnen und Vertretern der kaukasischen Rasse werden gleichsam alle bürgerlichen Tugenden zugesprochen: Fleiß, Wissensdurst, Beharrlichkeit, geistige und materielle Wünsche, die sie $\mathrm{zu}$ verwirklichen bestrebt sind. Demgegenüber werden allen anderen Rassen - begünstigt vom tropischen Klima - Sinnenfreude, die pure Lust, das Vergnügen, auch die Faulheit und Indolenz sowie die Begrenzung der eigenen Entwicklungsmöglichkeiten zugeschrieben, aber eben auch ein fröhliches, allzeit unbeschwertes Charakterchen bescheinigt. Eben hierin liegt, folgen wir dem französischen Anthropologen Claude Lévi-Strauss, der Rassismus: in der simplen und interessegeleiteten Übertragung biologischer in charakterliche und kulturelle Zuschreibungen.

Die Position Fredrika Bremers ist dabei überdeutlich. Gleichzeitig macht sie klar, dass sie in einem Land, in dem Sklaverei herrscht, nicht leben könne, dass für sie die Freiheit das absolute Gut ist, das zum Leben lebensnotwendig sei. Vieles im obigen Zitat wäre eines Kommentars wert, gerade auch das Licht, das auf die kulturellen Praktiken und Äußerungsformen der kubanischen Bevölkerung und insbesondere der Schwarzen geworfen wird. Insbesondere Musik und Tanz werden hierbei für Kuba stellvertretend aufgeführt. Der hermeneutische Zirkel hat sich auf diese Weise über einer Insel geschlossen, die von Beginn an beides war: irdisches Paradies und Hölle, Ort der freien Schwarzen und der Versklavung, der afrikanischen Trommeln und der französischen Mode. Aber alles erscheint im Lichte eines europäischen Wertesystems, das auf die gesamte Erde übertragen wird.

Kubas Bild ist von Ambivalenz, zugleich aber auch von größter Heterogenität geprägt. Erstaunlich ist, dass ein wirkliches breit angelegtes Gemälde einer künftigen Entwicklung auf Kuba nicht auftauchen will: Wir lassen die Insel auf Nimmerwiedersehen zurück, hören keine Prophezeiung hinsichtlich ihres 
künftigen Werdegangs oder ihrer zukünftigen Bestimmung. Sakrale Deutungselemente werden auf die Insel projiziert, ganz im Sinne des 19. Jahrhunderts, in dem eine Desakralisierung des Sakralen und eine Resakralisierung des NichtSakralen stattfanden. Die Sichtweise Fredrika Bremers ist eindeutig von den Einschätzungen ihrer Gastgeber, der weißen internationalisierten Familien der Oberschicht, wenn auch nicht von der spanischen Kolonialverwaltung, geprägt. Ihre Kritik an der Sklaverei geht nicht in eine generelle Kritik einer stark hierarchisierten Gesellschaft über, sondern versucht, einen Blick zu entwickeln, der diese Schichtung möglichst genau erkundet.

Eben hierin liegt die große Bedeutung Fredrika Bremers und ihrer Reisebriefe: Die Präzision ihrer Beobachtungen zum Zeitpunkt ihres Besuches und der Versuch, ein Gesamtbild der Insel aus einer subjektiven Sicht zu entwerfen, die auf einer Empathie beruht und ihre eigene Sichtweise - ganz im Sinne der Romantik - aus Kontrasten aufbaut. Ein letztes Mal wird der Blick von oben auf die Stadt geworfen, ein Blick wie aus größerer Höhe, von höherer Warte aus, der noch einmal alles zusammenfasst, und doch nicht mit dem Gegenstand verschmilzt. Denn die Bewegung der Rückreise schreibt sich unmittelbar in die Wahrnehmung des Reisegegenstandes und in dessen Konturierung ein. Fredrika Bremer kehrt wieder in ihre eigene Welt zurück. 